\title{
Single Event Upset Studies with the Optical Links of the ATLAS SemiConductor Tracker
}

\author{
J.D. Dowell, R.J. Homer, G. Mahout, P. Jovanovic \\ The University of Birmingham, Great Britain. \\ I-M. Gregor \\ Wuppertal University, Germany. \\ R.L. Wastie, A.R. Weidbergi \\ Oxford University, Great Britain. \\ J. K. Troska ${ }^{\text {ii }}$, D. J. White \\ Rutherford Appleton Laboratory, Great Britain.
}

\begin{abstract}
Studies have been performed of Single Event Upsets in the ATLAS SemiConductor Tracker optical links system. The measurements were made using low energy neutrons, and pion and proton beams in the momentum range 300 to $465 \mathrm{MeV} / \mathrm{c}$. The implications for the operation of the system in ATLAS during high luminosity LHC operation are discussed.
\end{abstract}

Keywords: LHC; Data transmission; ASICs; optoelectronics; photodiode; Radiation tolerance; Single Event Upset.

\section{Introduction}

Optical links will be used in the ATLAS SemiConductor Tracker (SCT)[1] to transmit data from the silicon strip detector modules to the off-detector electronics and to distribute the Timing, Trigger and Control (TTC) data from the counting room to the front-end electronics[2]. During the operation of the SCT at the Large Hadron Collider (LHC), all the on-detector components will be exposed to large fluences of charged and neutral particles. The SCT on-detector components have been designed to be sufficiently radiation tolerant to survive 10 years of LHC operation. The results of the radiation hardness studies of the ASICs, epitaxial silicon PIN photodiodes, VCSELs $^{\mathrm{iii}}$ and fibres are described in previous publications[3,4,5,6,7,8]. As well as surviving the large fluences, the SCT optical links have to operate reliably whilst being exposed to a very high flux of charged and neutral particles. These high fluxes of particles can cause Single Event Upsets (SEU) and Single Event Burn-out. This paper describes the SEU studies with the SCT optical links.

\footnotetext{
${ }^{\text {i }}$ Corresponding author. Email: t.weidberg1@physics.ox.ac.uk

ii Now at EP Division, CERN, Geneva 23, Switzerland.

${ }^{\text {iii }}$ Vertical Cavity Surface Emitting Lasers
} 
Many authors have reported SEU studies in a variety of contexts. Huhtinen and Faccio[12] describe a computational method to evaluate SEU rates in an accelerator environment that is relevant to the present study. Their paper contains references to earlier related work. More recently Andrieux et al.[9] and Faccio et al.[17] have reported SEU studies for optical links to be used at the LHC.

The effects of radiation on the VCSELs, PIN diodes, on-detector ASICs and fibre are described in references $[3,4,5,6]$. The most relevant radiation induced effect for the SEU studies is the decrease in responsivity of the silicon PIN diodes. The unirradiated PIN diodes have an average responsivity of $0.48 \mathrm{~A} / \mathrm{W}$, and this decreases rapidly with irradiation to $0.32 \mathrm{~A} / \mathrm{W}$ [5]. However, the responsivity remains constant with increasing radiation and shows no further decrease up to a fluence of $10^{15} 1 \mathrm{MeV}$ $\mathrm{n}_{\mathrm{eq}} / \mathrm{cm}^{2}$, which is a factor of 3 greater than the maximum expected for the SCT during ATLAS operation[1].

An overview of the SCT links system is given in Section 2. The possible sources of SEU in the SCT links system are discussed in Section 3. The expected ATLAS radiation environment and the facilities used to simulate this environment are described in Sections 4 and 5. The results of the SEU studies are given in Section 6 and the implications for ATLAS operation are described in Section 7. Finally some conclusions are drawn in Section 8.

\section{SCT Links System}

The SCT optical links are based on VCSELs and epitaxial silicon PIN diodes operating at a wavelength of $850 \mathrm{~nm}$. There will be one opto-package for each of the 4088 SCT detector modules, where each detector module contains 1536 silicon strips. Each of these opto-packages will contain two VCSELs for the data links and one PIN diode for the TTC link (see Figure 1). 


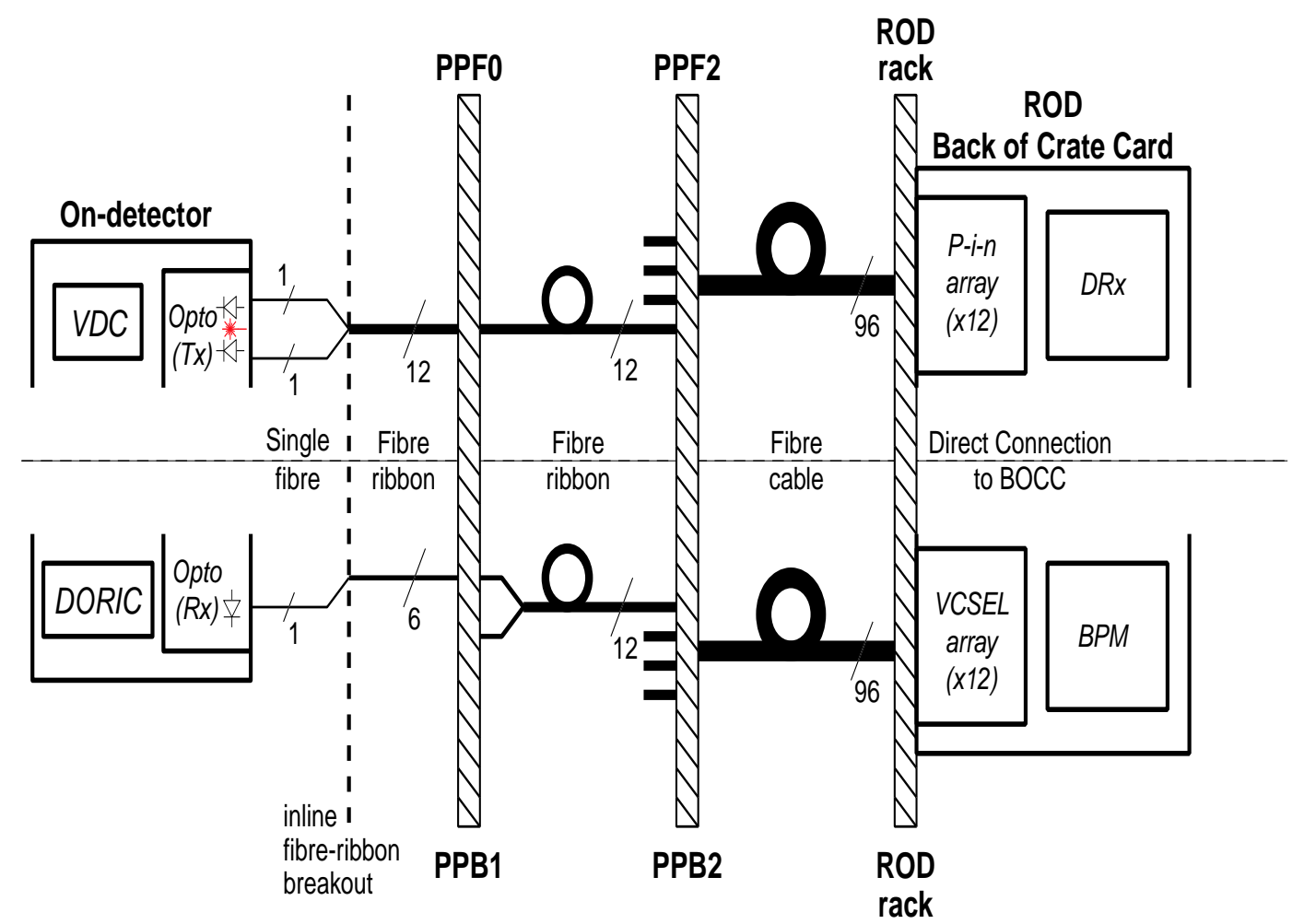

Figure 1 SCT Links architecture. PPB1, PPF0, PPB2, PPF2 refer to fibre patch panels.

The opto-packages are pig-tailed with multi-mode radiation-hard fibres[8]. The data from the SCT front end modules are read out serially via the VDC ASIC[5]. The data link uses an NRZ data format and the minimum fibre coupled power will be $300 \mu \mathrm{W}$. The off-detector opto-electronics for the data links consists of 12 way arrays of silicon PIN diodes to receive the optical signals and the DRX-12 ASIC[10] to amplify and discriminate the resulting electrical signals.

The TTC links use BiPhase Mark encoding to send the $40 \mathrm{MHz}$ bunch crossing (BC) signal and the $40 \mathrm{Mbits} / \mathrm{s}$ control data stream for each module down the same fibre. For the TTC links, the off-detector opto-electronics consists of the BPM12 ASICs[11] and 12 way VCSEL arrays. The BPM12 performs the BiPhase Mark encoding and drives the VCSELs. The TTC optical signal is converted to an electrical signal by the PIN diode in the on-detector opto-package and the resulting electrical signal is decoded by the DORIC4A ASIC to produce the recovered the BC clock and control data signals. The minimum fibre coupled power from these VCSELs is $1 \mathrm{~mW}$. Allowing for the maximum losses in the fibres, the connectors and the responsivity of radiation damaged silicon PIN diodes, the minimum mean current in the PIN diodes will be $75 \mu \mathrm{A}$. The specifications for the DORIC4A ASIC[3] require it to work down to a mean input current of $30 \mu \mathrm{A}$.

In order to minimise the SEU effects, the TTC system will always be operated at the maximum possible value of the mean PIN diode current. However in order to understand the origin of the SEU effects, the value was varied in this study. 


\subsection{Bit Error Rate Specifications}

Given the large expected optical signal used for the data links, the expected Bit Error Rate (BER) in the absence of radiation cannot easily be measured. For the data links a standard industry specification of BER of less than $10^{-9}$ is used. A BER of $10^{-9}$ would result in a negligible loss of silicon strip data compared to the expected inefficiency in the silicon detectors and electronics, which is of the order of $1 \%$. The same specification for the BER is used for the TTC links. Bit errors in the TTC link can cause an SCT module to fail to recognise a first level trigger (L1) signal. This will cause subsequent triggers for this module to be out of synchronisation with the rest of the system. Therefore valid data can only be obtained after a soft reset command is issued which resets the L1 counters on the front end SCT ASICs[1]. If such resets were to be issued to the SCT at a frequency of $1 \mathrm{~Hz}$, the resulting deadtime would be of the order of $0.1 \%$. With a BER of $10^{-9}$, the fraction of lost data from modules which have lost synchronisation, would be of the order of $0.01 \%$. Therefore the degradation in the SCT performance resulting from a BER of $10^{-9}$ in the TTC links would also be negligible.

\section{Sources of Single Event Upsets}

The passage of high energy particles through the optoelectronics and the ASICs can create problems. The deposition of a sufficiently large amount of energy in a transistor can cause a bit to flip and therefore create a bit error in the system. Apart from these Single Event Upsets (SEU), hard errors can occur in some systems, which would require a reset to be issued before the system would function again. A possible example of such a hard error in the ATLAS SCT links system would be if the Delay Locked Loop (DLL) in the DORIC4A[3] ASIC mis-locked. However the DORIC4A was designed to minimise the possibility of such errors occurring, so this is not expected to be a significant problem. An even more serious effect can happen if an error can cause a circuit to go into a state where it draws so much current that the ASIC is damaged (Single Event Burn-out, SEB). The ASICs used were designed with protection resistors so that SEB is not expected to occur. The ASICs were also designed to minimise SEU effects by operating at relatively high signal levels. They use only bipolar transistors so effects such as latch-up which can occur in CMOS circuits, would not be expected.

The parts of the system that are expected to be most sensitive to SEU effects are the PIN diode and the amplifier of the receiver ASIC, DORIC4A as these are the only parts of the system which are sensitive to relatively small signals. The active region of the PIN diode ${ }^{i}$ has a diameter of $350 \mu \mathrm{m}$ and a thickness of $15 \mu \mathrm{m}$, which is very much larger than the active region of the transistors in the DORIC4A amplifier. Hence the SEU effects are expected to occur dominantly in the PIN diode. The DORIC4A amplifier is ac coupled with a threshold close to zero. Therefore the minimum extra charge required to create a bit error will increase with increasing value of the mean current in the PIN diode, $\left\langle\mathrm{I}_{\mathrm{PIN}}\right\rangle$. Thus if the above hypothesis for the source of SEU is correct, the SEU rate should decrease with increasing value of $\left\langle\mathrm{I}_{\mathrm{PIN}}\right\rangle$. The

\footnotetext{
${ }^{\mathrm{i}}$ Centronic APEX10 Epitaxial silicon PIN diode.
} 
minimum energy deposition in the PIN diode required to create a SEU is in the few $\mathrm{MeV}$ range, which is much larger than the most probable energy deposition by Minimum Ionising Particles (MIPs) of $58 \mathrm{keV}$. Therefore MIPs are not expected to create SEU effects. However high energy particles can undergo nuclear interactions, which can produce strongly ionising heavy ions, which can deposit a few $\mathrm{MeV}$ of energy in a small volume[12]. The minimum energy deposition in the active volume of the PIN diode required to create a bit error was in the range 1 to $8 \mathrm{MeV}$ for the range of values of $\left\langle\mathrm{I}_{\mathrm{PIN}}\right\rangle$ used in these measurements. This therefore corresponds to a Linear Energy Transfer (LET) in the range $29 \mathrm{MeV} \mathrm{gm}^{-1} \mathrm{~cm}^{2}$ to $230 \mathrm{MeV} \mathrm{gm} \mathrm{gm}^{2}$.

\section{Radiation Environment}

The radiation received by the SCT will be dominated by primary and secondary particles from the proton-proton collisions. The expected fluxes of charged and neutral particles have been calculated for different positions in the SCT assuming that the LHC will operate at the high luminosity of $10^{34} \mathrm{~cm}^{-2} \mathrm{~s}^{-1}$ [1]. As discussed in Section 3 the dominant contributions to the SEU rates are expected to be from high energy hadrons. The fluxes of hadrons with kinetic energies greater than $20 \mathrm{MeV}$ in the barrel SCT are summarised in Table 1 below[1]. The fluxes expected for the forward SCT are in the same range.

Table 1 The expected flux of hadrons with energies greater than $20 \mathrm{MeV}$ for different positions in the SCT, during high luminosity LHC operation.

\begin{tabular}{|l|l|l|}
\hline Barrel layer & Radius $(\mathrm{cm})$ & $\begin{array}{l}\text { Hadron flux }(\mathrm{KE}>20 \mathrm{MeV}) \\
\left(\mathrm{cm}^{-2} \mathrm{~s}^{-1}\right)\end{array}$ \\
\hline 1 & 30 & $1.210^{6}$ \\
\hline 2 & 37 & $8.310^{5}$ \\
\hline 3 & 45 & $6.310^{5}$ \\
\hline 4 & 52 & $5.010^{5}$ \\
\hline
\end{tabular}

The charged particles are mainly pions with energy in the range $100-1000 \mathrm{MeV}$. The distribution in energy of the neutrons, peaks around $1 \mathrm{MeV}$ so that the neutrons make only a small contribution to the flux of hadrons with energies greater than $20 \mathrm{MeV}$.

\section{Irradiation Facilities}

\subsection{Minimum lonising Particles}

A $660 \mathrm{MBq}{ }^{90} \mathrm{Sr}$ source was used to provide electrons with kinetic energies of up 0.55 $\mathrm{MeV}$ to simulate the effect of Minimum Ionising Particles (MIPs). The flux of MIPs used was $4.310^{7} \mathrm{~cm}^{-2} \mathrm{~s}^{-1}$, which is a factor of over 20 higher than the highest flux expected for the SCT (see Table 1). 


\subsection{Neutrons}

The first tests with neutrons were performed at the Birmingham University Nuffield Cyclotron. Neutrons were created by the reaction $\mathrm{D}+\mathrm{Ag} \rightarrow \mathrm{n}+\mathrm{X}$. The primary $\mathrm{D}$ beam energy was $18 \mathrm{MeV}$ and the mean energy of the neutrons was $9 \mathrm{MeV}$. The flux for neutrons with energies greater than $7 \mathrm{MeV}$ was measured using aluminium foils to be $1.110^{6} \mathrm{~cm}^{-2} \mathrm{~s}^{-1}$. Further tests with neutrons were performed at the National Physical Laboratory (NPL) in London. Neutrons were created from $150 \mathrm{keV}$ D ions by the reaction $\mathrm{D}+\mathrm{T} \rightarrow \mathrm{n}+\alpha$. This created a nearly mono-energetic beam of neutrons, with an energy of $14.7 \mathrm{MeV}$. The fluxes were determined from the alpha particle yields measured with a silicon surface barrier detector[13,14]. The highest flux used was $510^{7} \mathrm{~cm}^{-2} \mathrm{~s}^{-1}$.

\subsection{Pions and Protons}

Beams of pions and protons were created at the $\pi \mathrm{M} 1$ beam line at the Paul Scherrer Institute (PSI), Switzerland. A primary proton beam with a kinetic energy of $590 \mathrm{MeV}$ from the Ring Cyclotron[15] impinged on a target. Mixed beams of pions and protons were used. The two dipole magnets of the beam line were used to select the momenta in the range 300 to $465 \mathrm{MeV} / \mathrm{c}$.

The beam profile was measured with two multi-wire proportional chambers (MWPCs) and the fluxes were measured with two scintillation counters (S1 and S2), as shown in Figure 2 below.

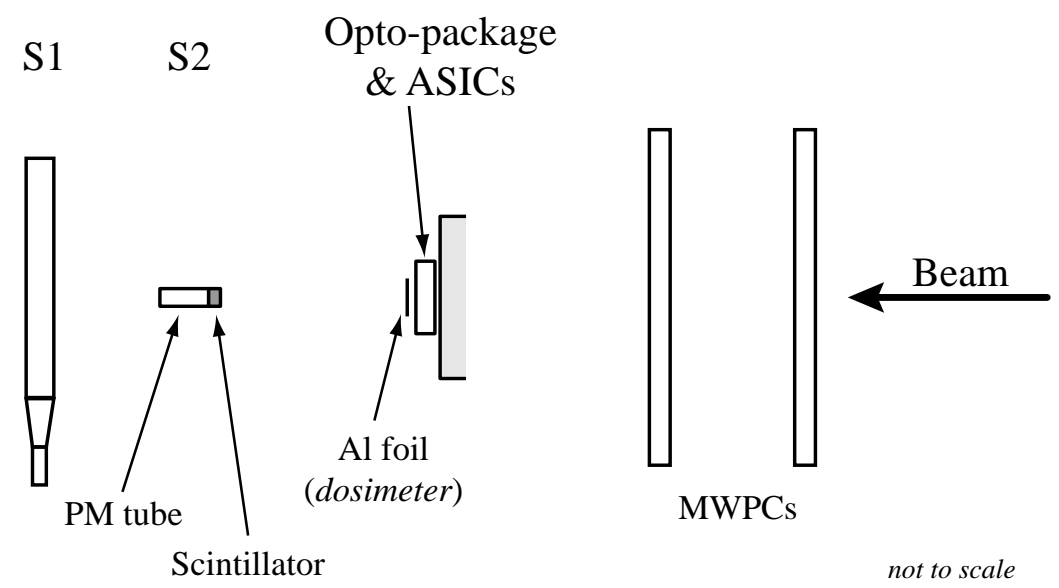

Figure 2 Schematic view of the layout of the scintillation counters, wire chambers and DUT in the target area of the PSI $\pi$ M1 beam line.

The MWPCs had a 2mm wire spacing: one measured the profile in the horizontal direction and the other in the vertical. The counter S2 contained a circular piece of scintillator of $2 \mathrm{~mm}$ diameter and $1 \mathrm{~mm}$ thickness. The scintillator was directly coupled with optical grease to a small photomultiplieri. The counter S1 was a conventional large area scintillation counter.

\footnotetext{
${ }^{\mathrm{i}}$ Hamamatsu R5600 Photomultiplier.
} 
Upstream slits were used to limit the beam flux during the operation of the MWPCs. At low beam fluxes the large scintillation counter S1 was used to trigger the MWPCs. Once the beam had been tuned and the profile measured, the high voltage for the MWPCs and the counter S1 were turned off and the slits opened up to produce the maximum flux. The beam profile was also measured using an $x-y$ stage to move the small scintillator counter $\mathrm{S} 2$ and measure the rate as function of horizontal and vertical position. The beam profiles measured with $\mathrm{S} 2$ were found to be in good agreement with those measured by the MWPCs. From the pulses observed on the oscilloscope from the counter S2, it was possible to distinguish pions from protons due to their very different energy loss in this momentum range. It was found that the beam consisted dominantly of pions for all momenta, except for the highest momentum used $(465 \mathrm{MeV} / \mathrm{c})$ where protons were dominant.

A photograph of the opto-flex cable with the opto-package and associated VDC and DORIC4A ASICS[3] mounted in the beam line is shown in Figure 3 below.

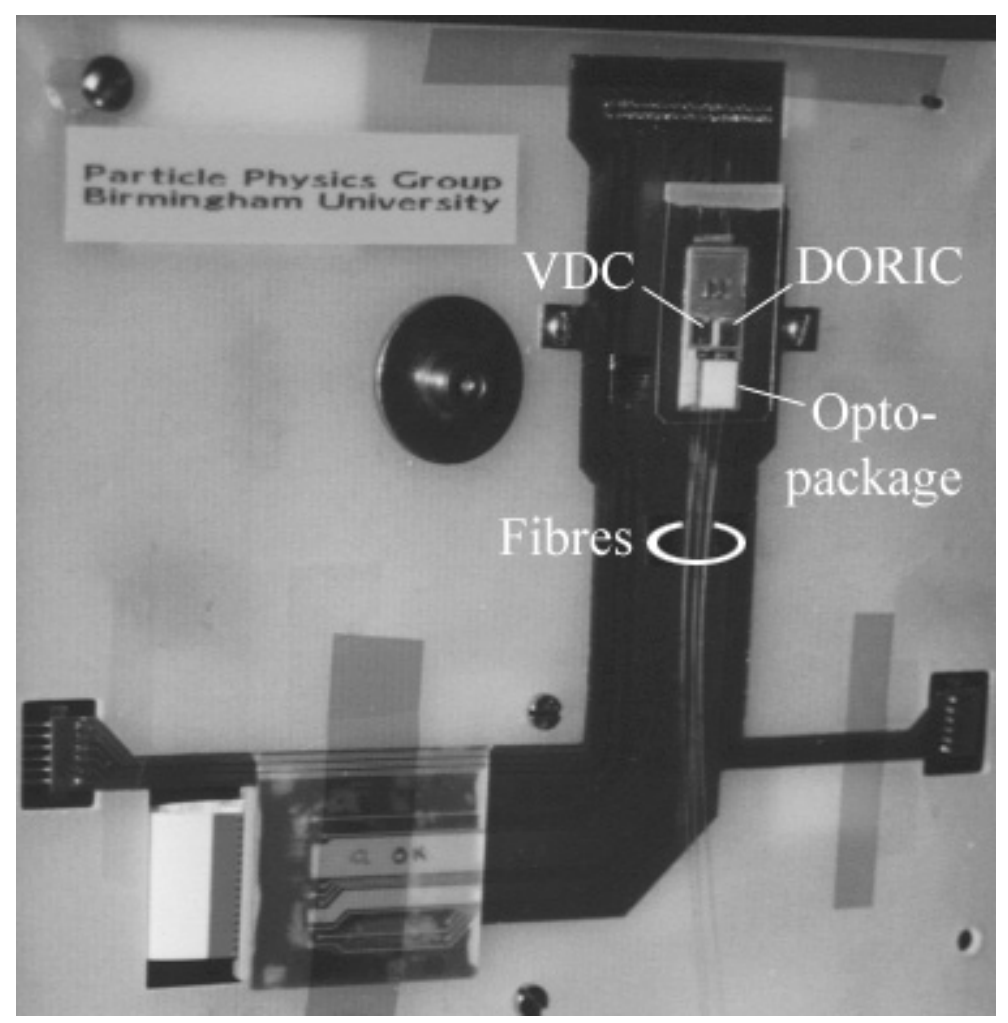

Figure 3 photograph of the opto-package and ASICs on the copper/kapton optoflex cable mounted on the support in the PSI beam line.

\subsubsection{Flux measurements}

The flux was determined from the rates measured in the counter S2. To first order the flux could be simply evaluated by dividing the measured rate by the area of the counter. Two corrections were applied to the raw value of the flux. First a simple program was used to correct for the deadtime of the discriminator which had been set to $100 \mathrm{~ns}$, allowing for the $20 \mathrm{~ns}$ bunch structure of the machine. Secondly the flux with the machine on was noted and a correction was made for the average on-time of the machine which could be evaluated as the primary currents in the machine were recorded continuously. Two cross checks of this procedure were performed. 
Firstly with the slits in the narrow position to limit the flux, the rates in the two counters S1 and S2 were measured. The counter S1 was large enough to fully contain the beam. The predicted ratio of the rates in S1 and S2 was calculated by fitting the measured beam profiles to a Gaussian distribution. The predicted ratio agreed with the measured ratio to within $10 \%$.

The second cross check of the flux measurements with the small counter was performed using aluminium foil activation measurements. The reaction $\pi+{ }^{27} \mathrm{Al} \rightarrow$ ${ }^{24} \mathrm{Na}+\mathrm{X}$ was used. Small aluminium foils were prepared and weighed and then attached to the back of the DUT. The foils were exposed in the beam and then removed. The resulting activation was measured using a $\mathrm{Ge}(\mathrm{Li})$ counter. The inclusive cross section for the production of $\mathrm{Na}^{24}$ was taken from ref. [16]. The results of this analysis are summarised in Table 2 below.

Table 2 Comparison of flux measurements from Al foils and scintillation counter.

\begin{tabular}{|l|l|}
\hline Flux determined from $\mathrm{Al}$ foil $\left(10^{6} \mathrm{~cm}^{-2} \mathrm{~s}^{-1}\right)$ & $4.25 \pm 0.25$ (stat) \pm 0.34 (syst) \\
\hline Flux determined from $\mathrm{S} 2\left(10^{6} \mathrm{~cm}^{-2} \mathrm{~s}^{-1}\right)$ & 4.71 \\
\hline
\end{tabular}

The agreement between the aluminium foil activation analysis and the scintillation counter for the flux was within $10 \%$ and consistent with the expected errors. From the good agreement of these two cross checks the systematic error on all flux measurements was taken to be $10 \%$. The statistical error on the flux measurements with the scintillation counter was negligible in comparison to the systematic error.

The beam momenta, flux and dominant particle composition for the different runs, are given in Table 3 below.

Table 3 Data for the different beams used at PSI.

\begin{tabular}{|l|l|l|}
\hline Momentum $(\mathrm{MeV} / \mathrm{c})$ & Flux $\left(10^{6} \mathrm{~cm}^{-2} \mathrm{~s}^{-1}\right)$ & Dominant particle type \\
\hline 300 & 2.9 & $\pi$ \\
\hline 350 & 4.7 & $\pi$ \\
\hline 350 & 57 & $\pi$ \\
\hline 405 & 5.0 & $\pi$ \\
\hline 405 & 120 & $\pi$ \\
\hline 465 & 120 & $\mathrm{p}$ \\
\hline
\end{tabular}

\section{SEU Measurements}

In this section the results of the SEU measurements are presented. The SEU effects were studied by measuring the Bit Error Rate (BER) in the links with and without beam. An increase in the BER with the beam on is interpreted as an SEU effect. The beams were used to uniformly irradiate all the on-detector opto-electronics, VCSELs, PIN diode and the DORIC4A and VDC ASICs. The hypothesis that the dominant source of SEU effects occurred in the PIN diode was investigated by measuring the 
SEU rate as a function of $\left\langle\mathrm{I}_{\mathrm{PIN}}\right\rangle$ (see Section 3). The BER was measured using the system described in ref. [2] and illustrated in Figure 4 below. The optical power of the off-detector VCSEL was varied by changing a DAC setting in the BiLED ASIC[2] and by the use of an optical attenuator. For the measurements performed with MIPs and neutrons the optical power was measured as a function of DAC value and the results are presented as a function of the optical power. This optical power can be converted into a current in the PIN diode if the responsivity is known. However the responsivity was varying during the measurements because of the irradiation (see Section 1). Therefore for the pion and proton measurements the current in the PIN was monitored directly by measuring the voltage drop across a series resistor in the PIN bias power supply.

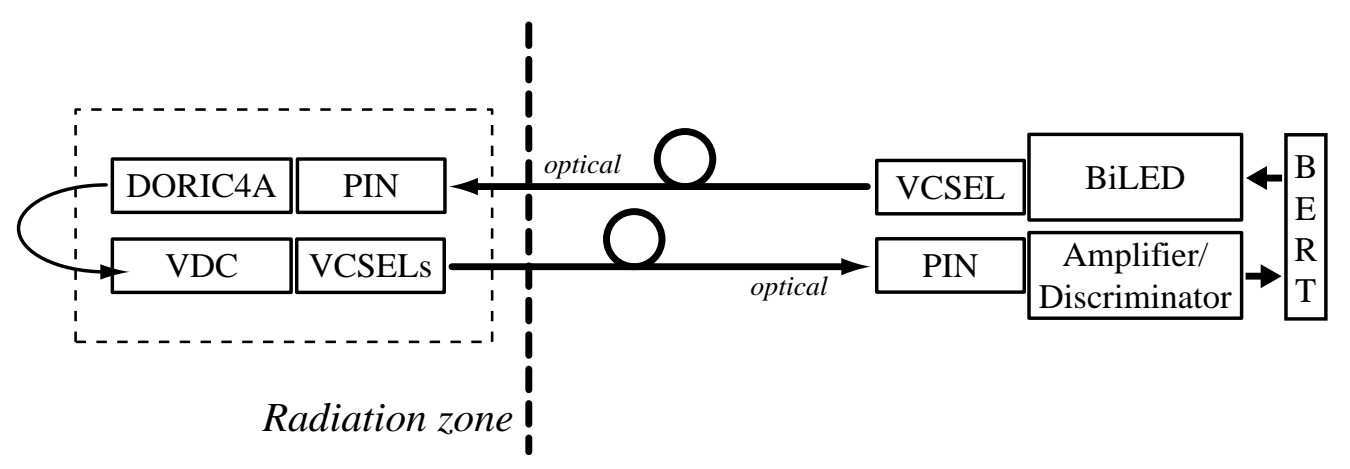

Figure 4 Schematic of the test system during SEU measurements. BERT= Bit Error Rate Tester.

A $32 \mathrm{k}$ deep pseudo-random number sequence was sent down the TTC link to the opto-package at a signal rate of $40 \mathrm{Mbits} / \mathrm{s}$. The resulting PIN diode signal was decoded by the DORIC4A ASIC and the command data were fed into one of the channels of the VDC ASIC. The VDC drove one of the VCSELs in the opto-package. The light from the opto-package was sent into a multi-mode fibre. The optical signal was detected by a PIN diode array and the electrical signal went into an amplifier/discriminator module[2]. The BER was measured by comparing the recovered data with the suitably delayed input data[2].

The opto-package containing the VCSELs and PIN diode and the associated ASICs were mounted perpendicular to the beam for the measurements described in this paper. Some earlier measurements showed that very heavily ionising particles, which are travelling parallel to the surface of the PIN diode can cause SEU effects by ionisation loss alone, i.e. without requiring a nuclear interaction. However in the ATLAS tracker the flux of sufficiently ionising particles travelling parallel to the surface of the PIN diode will be negligible. High energy charged or neutral particles can only cause SEU by creating heavily ionising particles in a nuclear interaction. Therefore the orientation of the opto-package to the beam is not expected to affect the SEU rate. This hypothesis is in agreement with the results of similar SEU measurements with the CMS tracker optical links[17].

\subsection{MIPs}

The BER was measured with the ${ }^{90} \mathrm{Sr}$ source as described in Section 5.1. With an input optical power of $500 \mu \mathrm{W}$ in the TTC link, no errors were detected in 12 hours 
and the resulting $90 \%$ c.l. upper limit on the BER rate was $1.310^{-12}$. This confirms the expectation (see Section 3) that MIPs do not produce a significant SEU rate.

\subsection{Neutrons}

From the measurements performed at the Birmingham Cyclotron, no significant difference in BER with beam on and beam off was found as shown in Figure 5 below and confirmed by longer runs.

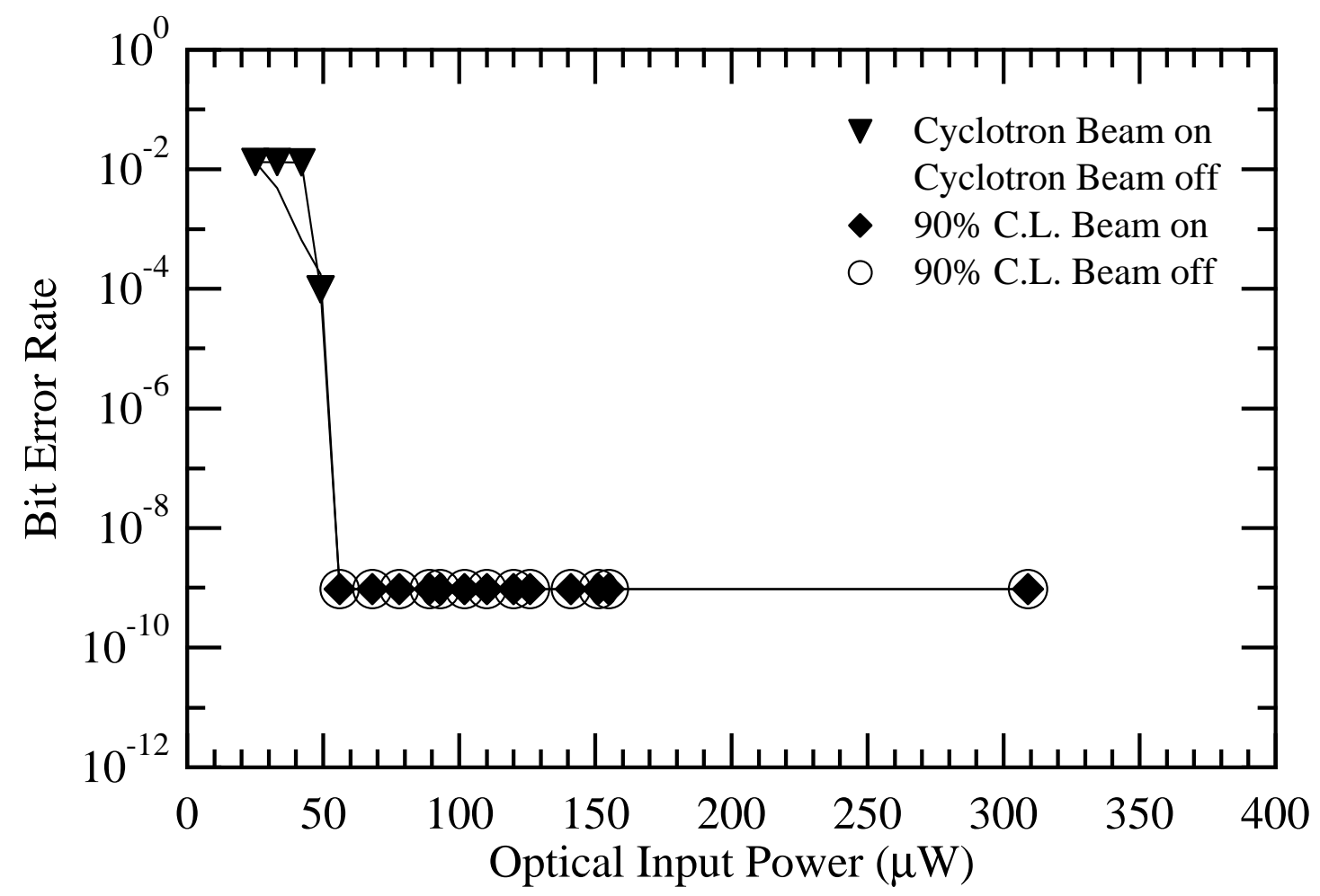

Figure 5 BER versus the mean optical power in the TTC. The data for amplitudes greater than $50 \mu \mathrm{W}$ correspond to upper limits at $90 \%$ confidence levels based on no observed errors in 60 second runs.

At an input optical power of $538 \mu \mathrm{W}$, the SEU rate was less than $4.110^{-11}$ at the $90 \%$ confidence level.

The measurements performed at NPL with higher energy and higher flux neutrons did show significant SEU effects. This is illustrated in Figure 6 below which shows the BER scans with and without beam. 


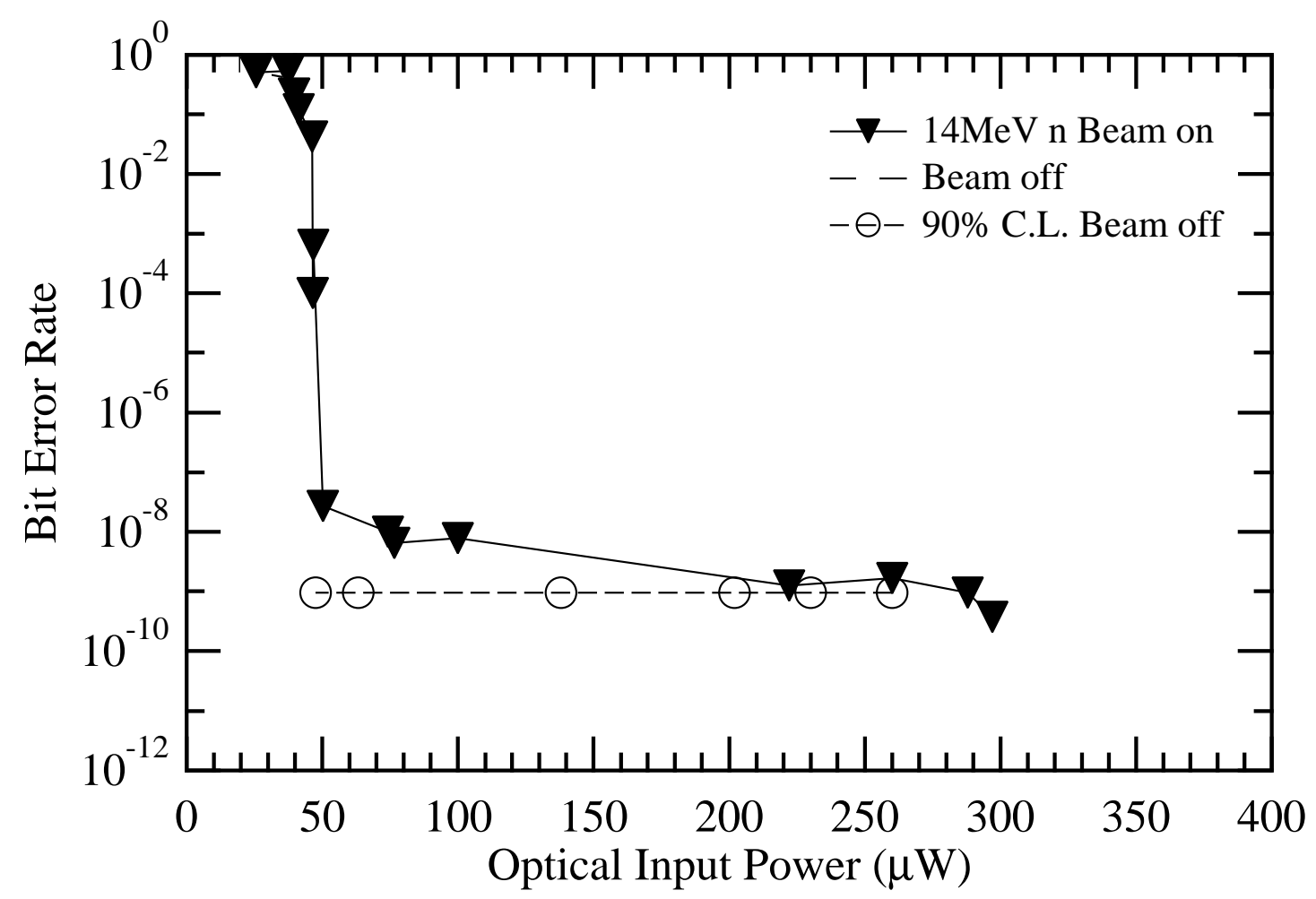

Figure 6 BER scans with beam off and a $14 \mathrm{MeV}$ neutron beam.

From a comparison of BER scans with beam on and beam off, there is clear evidence for significant SEU effects. Unfortunately the values of the PIN currents were not monitored during these measurements. Therefore the measurements can only be used to give a qualitative indication that SEU effects can occur as the systematic uncertainty is too large to use these measurements for any quantitative analysis. However the results confirm the hypothesis (see Section 3) that low energy neutrons do not produce significant SEU rates, provided the value of $\left\langle\mathrm{I}_{\mathrm{PIN}}\right\rangle$ is sufficiently large.

\subsection{Pions and Protons}

The result of a BER scan versus $\left\langle\mathrm{I}_{\mathrm{PIN}}\right\rangle$ for beam off data is compared with similar scans with beam on in Figure 7 below. The measurement durations varied between 1 minute at low values of $\left\langle\mathrm{I}_{\text {PIN }}\right\rangle$ to 1 to 6 hours at the higher values of $\left\langle\mathrm{I}_{\text {PIN }}\right\rangle$. 


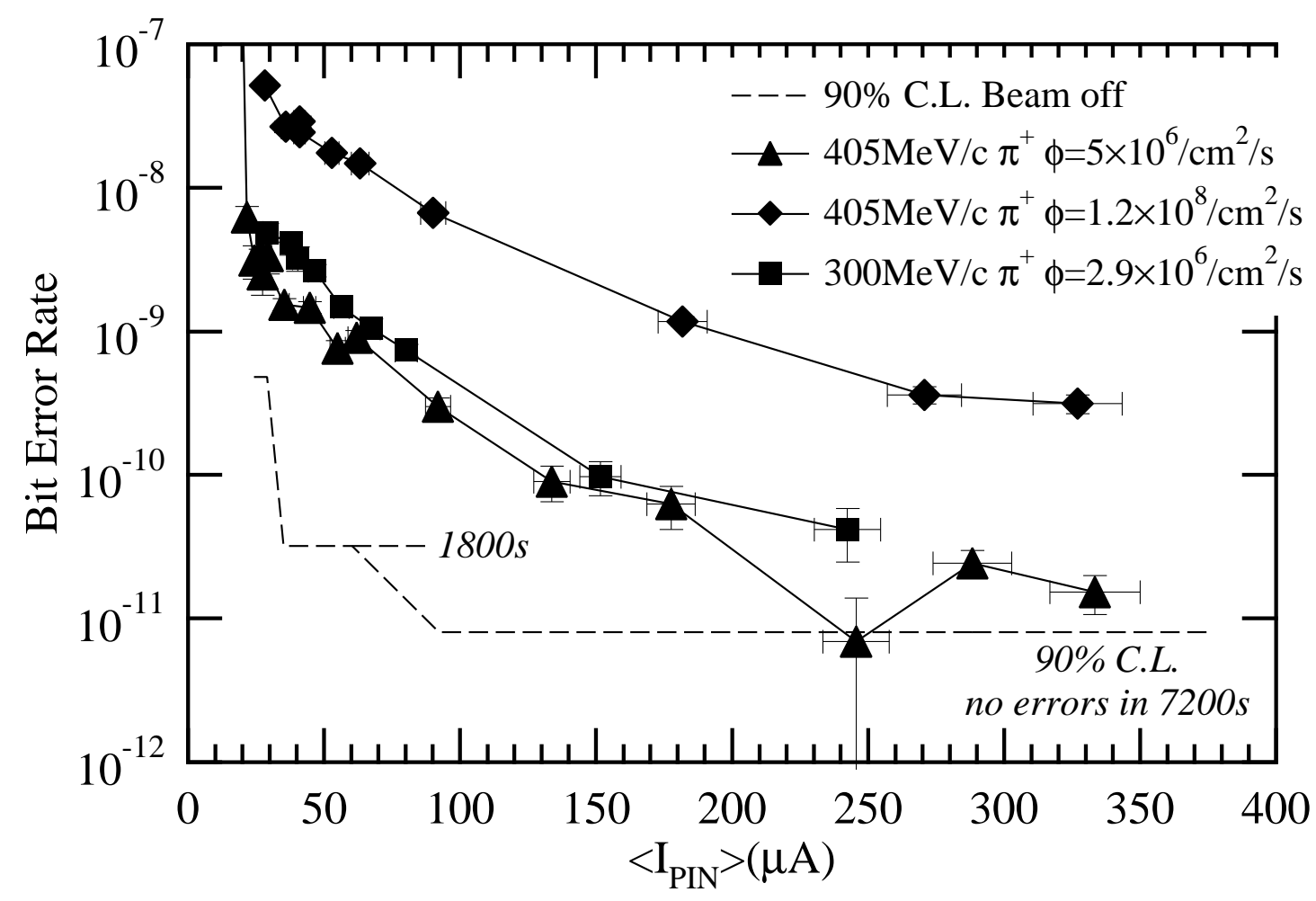

Figure 7 BER scans versus $<I_{\text {PIN }}>$ for beam off and different beam momenta and fluxes. Note that the symbols for the no beam data correspond to no errors and are $90 \%$ confidence level upper limits.

With beam off there were no errors for values of $\left\langle\mathrm{I}_{\mathrm{PIN}}\right\rangle$ greater than $20 \mu \mathrm{A}$, whereas there is a significant BER for this range of $\left\langle\mathrm{I}_{\mathrm{PIN}}\right\rangle$ with beam on. Therefore the BER in this range of $\left\langle\mathrm{I}_{\mathrm{PIN}}\right\rangle$ is due to SEU effects. No mis-lock of the DLL in DORIC4A occurred at any time.

The SEU induced BER decreases rapidly with increasing $\left\langle\mathrm{I}_{\mathrm{PIN}}\right\rangle$ as expected, which confirms the hypothesis that the dominant source of SEU effects is energy deposition in either the PIN diode or the transistors of the amplifier in DORIC4A. In a similar SEU study in CMS optical links, it was experimentally established that the dominant SEU effect was occurring in the PIN diode, not the amplifier[17]. The assumption that the SEU are occurring in the PIN diode will be used in this analysis. From the data taken with two different fluxes at the same beam momenta of $405 \mathrm{MeV} / \mathrm{c}$, the SEU rate scales linearly with flux as expected. In order to compare the data for the different beam momenta and fluxes, F, it is therefore convenient to define an SEU cross section by

$\sigma_{S E U}=N_{\text {ERRORs }} /(F t)$

where $\mathrm{N}_{\text {ERRORS }}$ are the number of errors occurring in time t. The plot of $\sigma_{\mathrm{SEU}}$ versus $\left\langle\mathrm{I}_{\mathrm{PIN}}\right\rangle$ is shown in Figure 8 below. 


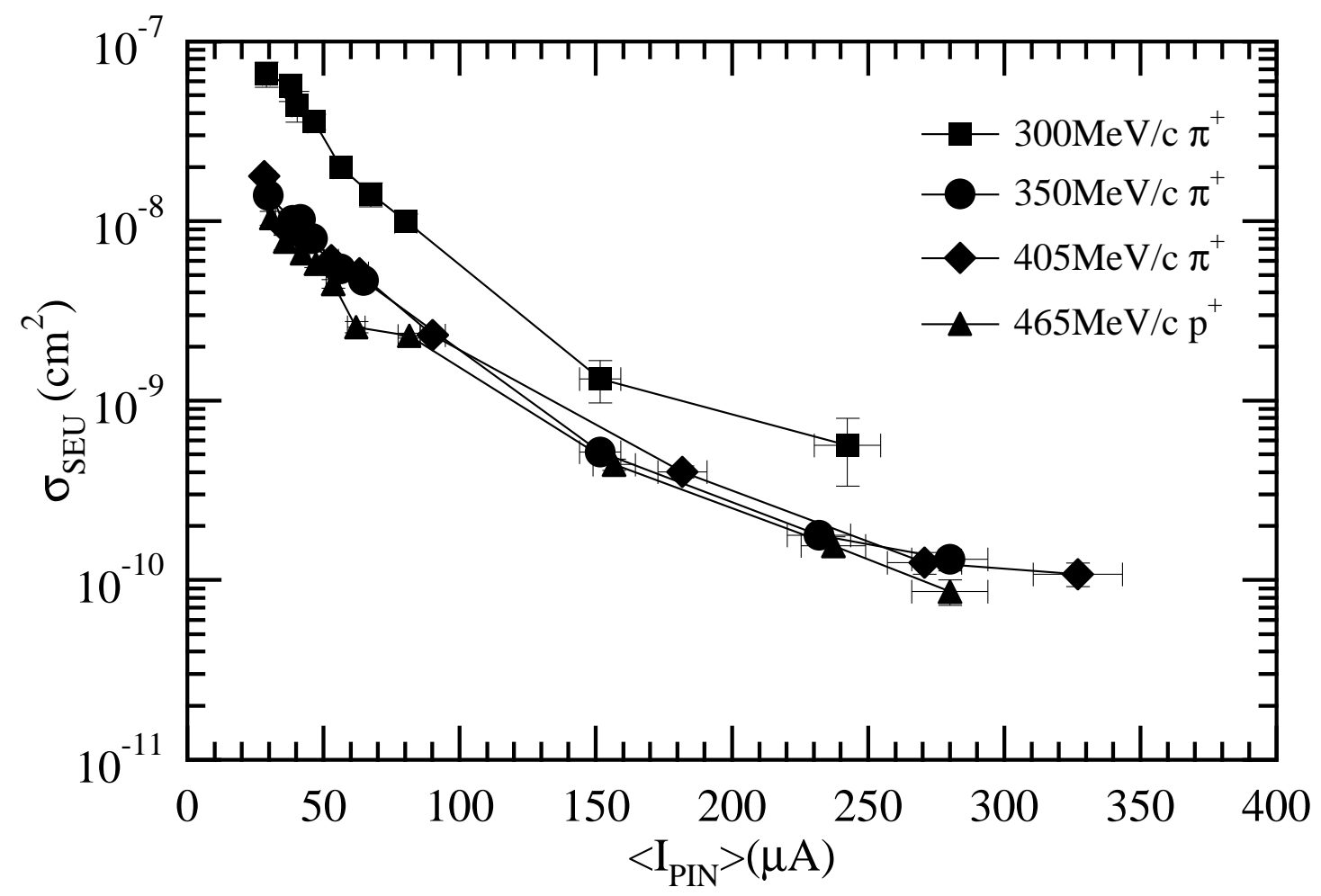

Figure 8 SEU Cross section versus $\left\langle I_{\text {PIN }}>\right.$ for different beam momenta.

The data for the different particle momenta and type all look very similar, apart from the $300 \mathrm{MeV} / \mathrm{c}$ data which show a significantly higher value of $\sigma_{\mathrm{SEU}}$ at the same values of $\left\langle\mathrm{I}_{\mathrm{PIN}}\right\rangle$. Enhanced damage in silicon diodes at the peak of the $\Delta$ resonance has been observed by Aarnio et al.[18] so such an effect is not unexpected.

In order to facilitate comparisons of this SEU cross section data with any future calculations that may be performed along the lines of ref. [12], it is convenient to convert the value of $\left\langle\mathrm{I}_{\mathrm{PIN}}\right\rangle$ into an equivalent energy deposition in the PIN diode $\left(\mathrm{E}_{\mathrm{min}}\right)$. Assuming a simple model for the DORIC4A input amplifier as being equivalent to an $\mathrm{RC}$ shaper, then the relation between $\mathrm{E}_{\min }$ and $\left\langle\mathrm{I}_{\mathrm{PIN}}\right\rangle$ is given by

$$
E_{M I N}=\left(I_{P I N}+I_{h}\right) \frac{E_{e h}}{e \omega_{0}}
$$

where $\mathrm{I}_{\mathrm{h}}$ is the current equivalent to the hysterisis in DORIC4A, $\mathrm{E}_{\mathrm{eh}}$ is the mean energy deposit required to create an electron-hole pair in silicon, $\omega_{0}$ is the inverse of the RC time constant of the DORIC4A amplifier and e is the magnitude of the electron charge. The values of the parameters used in equation (2) are given in Table 4 below.

Table 4 Values of parameters used in the model of the input amplifier for DORIC4A.

\begin{tabular}{|l|l|l|}
\hline \multicolumn{1}{|c|}{ Quantity } & Value & Units \\
\hline $\mathrm{I}_{\mathrm{h}}$ & 10.0 & $\mu \mathrm{A}$ \\
\hline $\mathrm{E}_{\mathrm{eh}}$ & 3.6 & $\mathrm{eV}$ \\
\hline$\omega_{0}$ & 1.0 & $(\mathrm{~ns})^{-1}$ \\
\hline
\end{tabular}


Using these values and equation (2), the data in Figure 8 were converted into the plot of $\sigma_{\mathrm{SEU}}$ versus $\mathrm{E}_{\min }$ shown in Figure 9 below. Since the SEU effects are simply due to energy deposition above a given threshold in the active region of the PIN diode, these data can be used for as a calibration of the simulations of the type used in ref. [12].

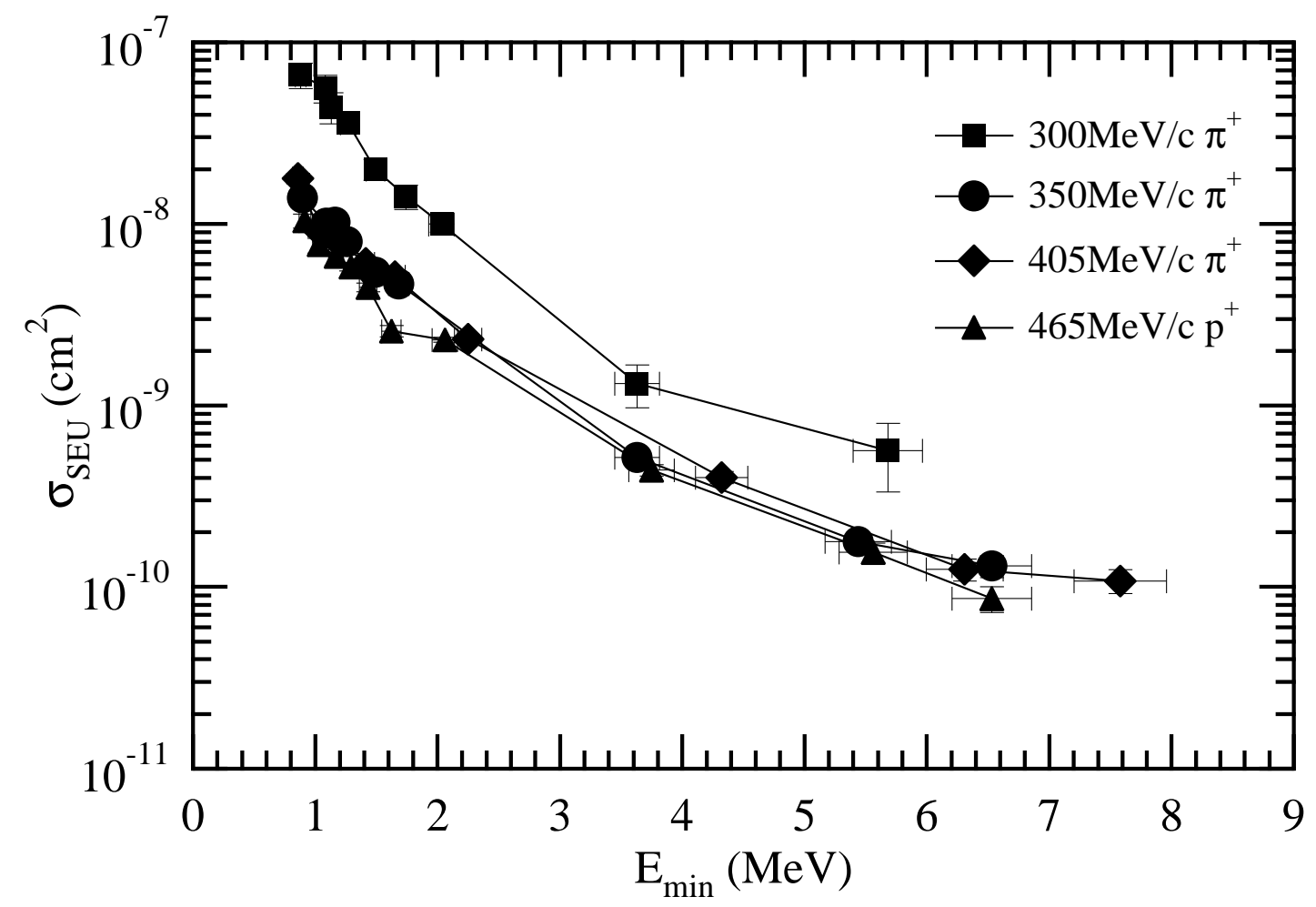

Figure 9 SEU cross section versus minimum energy deposition in the PIN diode for different beam momenta.

\section{Implications for ATLAS Operation}

The standard procedure for estimating SEU effects in ATLAS assumes that the SEU cross section is constant for energies above $20 \mathrm{MeV}$ [19]. However the data presented here are inconsistent with this hypothesis. In principle this data could be used to predict the SEU induced BER of the SCT TTC links during ATLAS operation by performing a convolution of $\sigma_{\mathrm{SEU}}$ with the expected particle spectrum:

$$
B E R=\frac{1}{D} \int \sigma_{S E U} \frac{d F}{d p} d p
$$

where $\mathrm{F}$ is the flux as a function of momentum, $\mathrm{p}$, and $\mathrm{D}$ is the data rate (40 Mbits/s). An accurate evaluation of this would require detailed simulations of the type performed in ref. [12] to predict the variation of $\sigma_{\mathrm{SEU}}$ with $\mathrm{p}$. However a conservative upper limit can be evaluated by using the $300 \mathrm{MeV} / \mathrm{c}$ data, which has the highest values of $\sigma_{\mathrm{SEU}}$. Combining these data with the minimum expected value of $\left\langle\mathrm{I}_{\mathrm{PIN}}\right\rangle$ of $75 \mu \mathrm{A}$ and the maximum flux expected in the SCT (see Table 1) gives an upper limit of $\mathrm{BER}=3.610^{-10}$, which is below the ATLAS SCT specification of $10^{-9}$. 


\section{Conclusions}

The results of SEU studies of the SCT optical links have been presented. As expected MIPs or low energy neutrons do not produce any significant SEU effects. Higher energy neutrons and charged particles do produce significant SEU effects. The data confirm the hypothesis that the SEU effects are due to an energy deposition in the PIN diode, which corresponds to a signal above the DORIC4A threshold. The SEU rate can be reduced by increasing the amplitude of the optical TTC signal. A conservative estimation of the SEU rate expected in ATLAS operation has shown that the expected BER during high luminosity operation is within the ATLAS SCT specifications.

\section{Acknowledgements}

We would like to thank Dr. Dieter Renker and Dr. Konrad Dieter (PSI) for invaluable help with the operation of the beam and for providing the beam monitoring equipment at PSI. We would also like to thank Dr. Kurt Gabathuler (PSI) for the use of the $\mathrm{Ge}(\mathrm{Li})$ spectrometer at PSI. We thank the PSI management for the allocation of beam time at PSI. We would also like to thank Dennis Grant, Roger Harris and Ian McGill at the University of Birmingham for technical support. Financial support from the UK Particle Physics and Astronomy Research Council is gratefully acknowledged.

\section{References}

1 ATLAS Inner Detector Technical Design Report, CERN/LHCC/97-16/17.

2 D.G. Charlton et al., System Test of Radiation Hard Optical Links for the ATLAS SemiConductor Tracker, Nucl. Inst. and Meth. A 443 (1999) 430.

3 D.J. White et al., Radiation Hardness Studies of the Front-end ASICs for the Optical Links of the ATLAS SemiConductor Tracker, Nucl. Instr. and Meth. A 457 (2001) 369.

4 J.D. Dowell et al., Irradiation tests of photodiodes for the ATLAS SCT readout, Nucl. Inst. and Meth. A 424 (1999) 483.

5 D.G. Charlton et. al., Radiation Hardness and Lifetime Studies of Photodiodes for the Optical Readout of the ATLAS SCT, Nucl. Instr. and Meth. A 456 (2001) 300.

$6 \mathrm{~J}$. Beringer et al., Radiation Hardness and Lifetime Studies of LEDs and VCSELs for the Optical Readout of the SCT, Nucl. Instr. and Meth. A 435 (1999) 375.

7 D.G. Charlton et al., Radiation Tests of Optical Link Components for the ATLAS

SCT, proceedings of the $4^{\text {th }}$ Workshop on Electronics for the LHC, Rome $21^{\text {st }}-25^{\text {th }}$

September 1998, CERN/LHCC/98-36.

8 G. Mahout et al., Irradiation Studies of Multimode Optical Fibres for use in ATLAS Front-end Links, Nucl. Instr. and Meth. A 446 (2000) 426.

9 M-L. Andrieux et al., Single-event upset studies of a high speed digital optical links, Nucl. Instr. And Meth. A 456 (2001) 342.

10 DRX-12 ASIC specifications are available on WWW at url: http://wwwpnp.physics.ox.ac.uk/ weidberg/drx_specs.pdf.

11 BPM-12 ASIC specifications are available on WWW at url: http://wwwpnp.physics.ox.ac.uk/ weidberg/bpm12_specs.pdf. 
12 M.Huhtinen and F. Faccio, Computational Method to estimate Single Event Upset rates in an accelerator environment. Nucl. Instr. and Meth. A 450 (2000) 155.

13 P. Kolkowski, NPL, private communication.

$14 \mathrm{~S}$. Shirato et al, Absolute determination of T-d neutron yields by the associated particle method, Nucl. Instr. and Meth. A 278 (1989) 477.

15 Information on the PSI ring cyclotron is available on WWW at url:

http://www1.psi.ch/www_gfa_hn/aea/.

16 B.J. Dropesky et al., Excitation functions for the production of ${ }^{18} \mathrm{~F}$ and ${ }^{24} \mathrm{Na}$ from $\mathrm{Al}$ and Si with fast pions. Phys. Rev. C 32 (1985) 1305.

$17 \mathrm{~F}$. Faccio et al., SEU tests of an $80 \mathrm{Mbit} / \mathrm{s}$ optical receiver. Proceedings of the $6^{\text {th }}$ Workshop on Electronics for LHC experiments, Cracow $11^{\text {th }}-15^{\text {th }}$ September, CERN 2000-010, 245-249.

18 P.A. Aarnio et al., Damage observed in silicon diodes after low energy pion irradiation, Nucl. Instr. Meth. A 360 (1995) 521.

19 Information on the ATLAS procedures is available on WWW at url:

http://atlas.web.cern.ch/Atlas/GROUPS/FRONTEND/radhard.htm\#Standard Test Methods 